\title{
Resultados de una EEC variando los grados de Corrosión y el tiempo de aplicación de la técnica
}

\author{
Results of an ECE Varying Degrees of Corrosion and Time of Application \\ of the Technique
}

\author{
Rodriguez-Reyna S.L. \\ Área Mecánica Eléctrica \\ Facultad de Ingeniería \\ Universidad Autónoma de San Luis Potosí \\ Correo: sandyreyna@uaslp.mx \\ Miranda-Vidales J.M. \\ Instituto de Metalurgia \\ Universidad Autónoma de San Luís Potosí \\ Correo:jmiranda@uaslp.mx
}

\author{
Espericueta-González D.E. \\ Área Mecánica Eléctrica \\ Facultad de Ingeniería, \\ Universidad Autónoma de San Luís Potosí \\ Correo:despericueta@uaslp.mx
}

Gaona-Tiburcio C.

Universidad Autónoma de Nuevo León - FIME - CIIIA

Correo: citlalli.gaona@gmail.com

Información del artículo: recibido: julio de 2010, reevaluado: septiembre de 2010, aceptado: enero de 2012

\section{Resumen}

El objetivo de la extracción electroquímica de cloruros (EEC) es remover los iones cloruro ( $\mathrm{Cl}$-) que se encuentran inmersos en el concreto, ya que se consideran causantes de acelerar el proceso de corrosión en la armadura de acero. La EEC es una técnica electroquímica en la cual los iones $\mathrm{Cl}$ - se transportan hacia el exterior del hormigón por medio de un campo eléctrico. En este trabajo se presentan los resultados obtenidos en muestras de mortero fabricadas en laboratorio. Las probetas fueron previamente contaminadas con $2 \% \mathrm{NaCl}$ con respecto al peso del cemento y colocadas dentro de una cámara a una HR en torno a 95\%, durante uno y sesenta días. Posteriormente a los morteros se les efectúo una EEC en tiempos de 15, 30 y 60 días de tratamiento. Los resultados obtenidos bajo estas condiciones muestran que al incrementar la duración de la EEC se obtiene una mayor cantidad de iones Cl- removidos del mortero. Cabe señalar que el grado de precorrosión de las armaduras no es una variable crítica en la efectividad de la técnica.

\section{Descriptores:}

- extracción electroquímica de cloruros

- acero de refuerzo

- mortero

- métodos electroquímicos de rehabilitación

- durabilidad

- corrosión 


\begin{abstract}
The objective of the Electrochemical Chloride Extraction (ECE) is to remove the chloride ions ( $\mathrm{Cl}$-) which are embedded in concrete, since these ions are considered to result in accelerating the process of corrosion in steel reinforcement. The $E C E$ is an electrochemical technique in which ions $\mathrm{Cl}$-are transported to the outside of the concrete by means of an electric field. In this paper we present the results of mortar samples made in the laboratory. The specimens were previously contaminated with $2 \% \mathrm{NaCl}$ by mass of cement, and stored in water curing tanks at constant laboratory of $95 \% \mathrm{RH}$ for one and sixty days. Afterward, mortars underwent an ECE in times of 15, 30 and 60 days of treatment. The results obtained under these conditions show that increasing the duration of the $E C E$ will get a higher amount of $\mathrm{Cl}$-ions removed from the mortar. It should be noted that the extent of the corroded reinforcement is a critical variable in the effectiveness of the technique.
\end{abstract}

\section{Keywords:}

- electrochemical chloride extraction

- reinforcing steel

- mortar

- electrochemical methods of rehabilitation

- durability

- corrosion

\section{Introducción}

La reducción de la durabilidad de las estructuras de hormigón por causa de la corrosión se debe a la pérdida del estado pasivo, ya sea en grandes áreas a causa de la carbonatación o localmente por efecto de los cloruros que sobrepasan una concentración crítica, llevando a las estructuras de hormigón a la disminución de su vida de servicio (Browne et al., 1983; Page y Treadaway, 1982). Existen métodos convencionales de reparación que exigen el reemplazo del hormigón contaminado y la limpieza a fondo de las armaduras, así como costos elevados de reparación (Biezma y San Cristobal, 2006). Esta situación ha llevado a la búsqueda de nuevas alternativas para reparar las estructuras corroídas. Un método alternativo de rehabilitación es la Extracción Electroquímica de Cloruros. Esta técnica consiste en utilizar una fuente de corriente, donde las varillas de acero se conectan a la terminal negativa (actuando como cátodos) y el electrodo externo a la terminal positiva (siendo el ánodo). Los iones cloruro con carga negativa migran a la superficie externa al aplicarse una densidad de corriente.

Una de las ventajas que ofrece la EEC consiste en la reducción de la concentración de $\mathrm{Cl}$ en el mortero por varias semanas, y no es de carácter destructivo, en comparación con las reparaciones convencionales (Lankart et al., 1975; Morrison et al., 1976).

En el presente trabajo se analizan los resultados obtenidos de una EEC en muestras de mortero, variando los grados de precorrosión, duración de la EEC y realizando análisis químico puntual de los porcentajes de $\mathrm{Cl}$ - en diferentes puntos del mortero después de la EEC.

\section{Procedimiento experimental}

\section{Materiales}

Para la fabricación de las muestras de mortero se utilizó cemento Pórtland CPC 30R, con una relación cemento/ arena/agua de $1 / 3 / 0.45$, las muestras de mortero fueron cilíndricas de $11 \mathrm{~cm}$ de diámetro y $10 \mathrm{~cm}$ de altura, dejando un recubrimiento de $3 \mathrm{~cm}$ a los lados y de $1 \mathrm{~cm}$ en la parte superior e inferior del cilindro. En cada una de las muestras cilíndricas de mortero se colocaron tres varillas de $0.7 \mathrm{~cm}$ de diámetro y de $8 \mathrm{~cm}$ de longitud, expuestas a la corrosión. Antes de colocar las varillas dentro de los cilindros, se descaparon con una solución de ácido clorhídrico a $50 \%$ con $4 \mathrm{~g} / 1$ de hexametilentetramina como inhibidor. Se colocó una cinta de aislar entre la parte superior desnuda de la varilla y la parte sumergida dentro del concreto, con la finalidad de evitar un ataque localizado por el fenómeno de aireación diferencial.

Las 8 muestras cilíndricas de mortero empleadas en la investigación se contaminaron desde su preparación con $2 \%$ de $\mathrm{NaCl}$ con relación al peso del cemento. Dos de las ocho muestras permanecieron en la cámara de humedad durante 140 días, estas muestras se utilizaron para observar el proceso de corrosión en los refuerzos de acero. A cada una de las seis muestras restantes se les realizó la EEC, una vez concluido su periodo de exposición en la cámara con una Humedad Relativa (HR) superior a 95\%. Los tiempos de exposición fueron de uno y de sesenta días para lograr grados de corrosión diferentes. La duración de las extracciones fueron de 15, 30 y 60 días, suministrando una densidad de co- 
rriente constante de $1 \mathrm{~A} / \mathrm{m}^{2}$ (sobre la superficie del acero). En la tabla 1 se presentan los datos característicos de las muestras utilizadas para el desarrollo experimental.

Tabla 1. Muestras utilizadas en la experimentación

\begin{tabular}{cccc}
\hline $\begin{array}{c}\text { Núm. } \\
\text { muestra }\end{array}$ & $\begin{array}{c}\text { Tipo de } \\
\text { Contaminante }\end{array}$ & $\begin{array}{c}\text { Tiempo previo a } \\
\text { la EEC } \\
\text { (días) }\end{array}$ & $\begin{array}{c}\text { Tiempo de la } \\
\text { EEC (días) }\end{array}$ \\
\hline $1 \mathrm{~A}$ & $2 \% \mathrm{NaCl}$ & 1 & 15 \\
$2 \mathrm{~A}$ & $2 \% \mathrm{NaCl}$ & 1 & 30 \\
$3 \mathrm{~A}$ & $2 \% \mathrm{NaCl}$ & 1 & 60 \\
$4 \mathrm{~A}$ & $2 \% \mathrm{NaCl}$ & 140 & SIN EEC \\
$5 \mathrm{~A}$ & $2 \% \mathrm{NaCl}$ & 140 & SIN EEC \\
$6 \mathrm{~A}$ & $2 \% \mathrm{NaCl}$ & 60 & 15 \\
$7 \mathrm{~A}$ & $2 \% \mathrm{NaCl}$ & 60 & 30 \\
$8 \mathrm{~A}$ & $2 \% \mathrm{NaCl}$ & 60 & 60 \\
\hline
\end{tabular}

\section{Ensayos electroquímicos}

Para las mediciones electroquímicas, dos de las tres varillas de acero introducidas en el cilindro de mortero se utilizaron como barras de refuerzo, y la tercera como contraelectrodo, siendo el área de exposición para el tratamiento de $17 \mathrm{~cm}^{2}$ en cada varilla. El comportamiento de los electrodos con y sin EEC, con respecto al tiempo, se siguió a través de la medida de potencial de corrosión, $\mathrm{E}_{\text {corr }}$ y la resistencia de polarización, Rp (con el fin de calcular la velocidad de corrosión a partir de la ecuación de Stern y Geary (1957). La Rp permitió conocer el estado pasivo o activo de los refuerzos. El electrodo de referencia empleado en las medidas de $E_{\text {corr }}$ y Rp fue el electrodo saturado de calomelanos (ESC). En la figura 1 se muestran los prototipos y las mallas de titanio conectadas a la fuente poder.

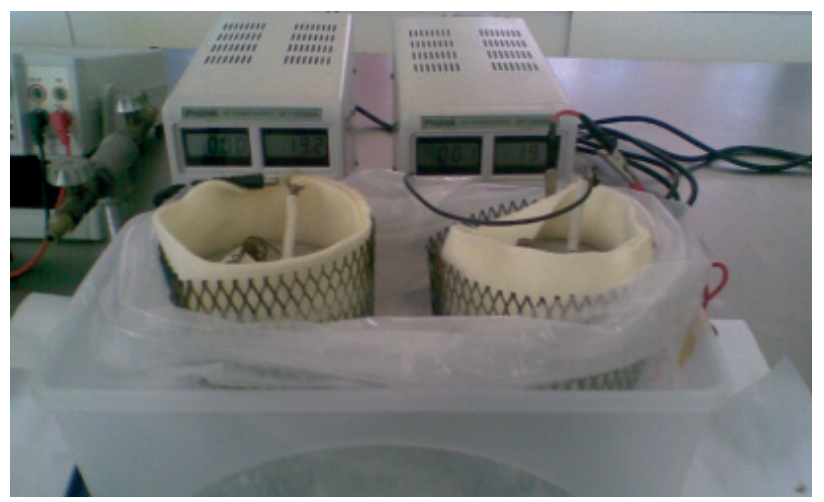

Figura1. Prototipos y mallas de titanio conectadas a la fuente de poder para efectuar la EEC

\section{Discusión y resultados}

En la figura 2 se muestran los porcentajes de cloruros removidos del mortero, variando en el grado de precorrosion y la duración de las extracciones. En los cilindros $1 \mathrm{~A}$ y $6 \mathrm{~A}$, que tuvieron 15 días de EEC, sus eficiencias fueron de 21 y $19 \%$, respectivamente. Las muestras 2A y 7A, con 30 días de extracción, lograron eficiencias de 25 y $27 \%$. Finalmente, las muestras $3 \mathrm{~A}$ y $8 \mathrm{~A}$, con sesenta días de tratamiento, lograron la eficiencia más alta de aproximadamente $36 \%$. Se puede observar que las muestras con distintos tiempos de precorrosión y con los mismos tiempos de extracción, presentan eficiencias semejantes en el tratamiento; lo que supondría que el grado de precorrosión no afecta directamente con la eficiencia de la técnica. Asimismo, los porcentajes de cloruros removidos del mortero aumentan conforme se incrementa la duración de la EEC.

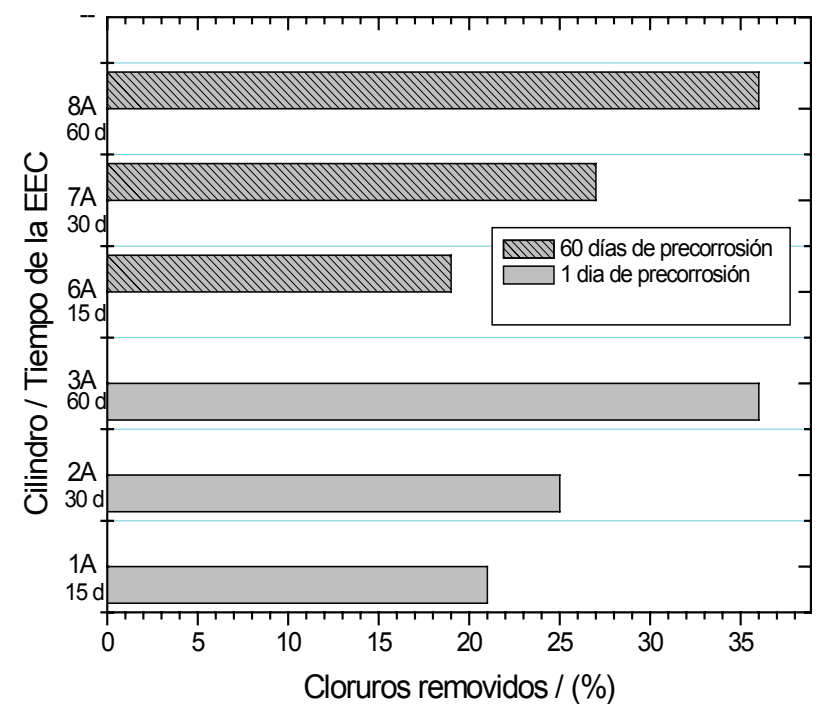

Figura 2. Porcentaje de cloruros removidos en muestras de mortero después de realizar EEC durante 15, 30 y 60 días

Otros autores como Orellan et al. (2004) removieron hasta $40 \%$ de cloruros después de 7 semanas de tratamiento, aplicando la misma densidad de corriente.

En la figura 3 se presenta la evolución de los potenciales en función del tiempo, antes, durante y después de las EEC, con quince (3a) y treinta días de tratamiento (3b). Se observa que antes de iniciar las extracciones los potenciales se situaban en valores negativos de $-430 \mathrm{mV}$, de acuerdo con la norma ASTM 876, estos potenciales sitúan a los refuerzos de acero en una zona de alto riesgo, con probabilidades $\geq 90 \%$ de que ocurra corrosión. Durante las extracciones estos valores se dispararon 
hasta alcanzar los $-1000 \mathrm{mV}$, debido a una polarización ocurrida por el tratamiento. Otros autores como Fajardo et al. (2006) trabajaron con cilindros de concreto previamente contaminados con $1 \% \mathrm{Cl}^{-}$, con respecto a la masa de cemento, aplicando una densidad de corriente de $1 \mathrm{~A} / \mathrm{m}^{2}$ encontraron que al medir los potenciales durante el tratamiento, las armaduras de acero presentaron una polarización y los valores de $\mathrm{E}_{\text {corr }}$ se situaron en torno a $-1050 \mathrm{mV}$. De acuerdo con Green et al. (1993), la disponibilidad de oxigeno es uno de los parámetros de control de la despolarización del refuerzo de acero; entre más rápido llegue a la superficie del acero, más rápido encuentra su estado de equilibrio. Sin embargo, los resultados demostraron lo contrario.

Un punto a resaltar en la investigación es el tiempo que tardaron las muestras en estabilizar sus potenciales, lo que dependió de la duración de la técnica. Entre más tiempo duraban las EEC, más tiempo tardaban los potenciales en estabilizarse. En general, los potenciales alcanzaron valores más positivos, situándolos en - $250 \mathrm{mV}$, siendo una zona incierta de corrosión como lo señala la norma ASTM 876. A pesar de que los valores fueron más positivos después del tratamiento, esto no podría garantizar que las varillas de acero se despasivaran.

En la figura 4 se presentan los $\mathrm{E}_{\text {corr }}$ de las armaduras de acero antes, durante y después de la extracción realizada durante sesenta días. Adicionalmente se agregaron a la gráfica los valores de los potenciales de las varillas de acero cuando las muestras no fueron sometidas a ningún tratamiento y tampoco fueron contamina-

a)

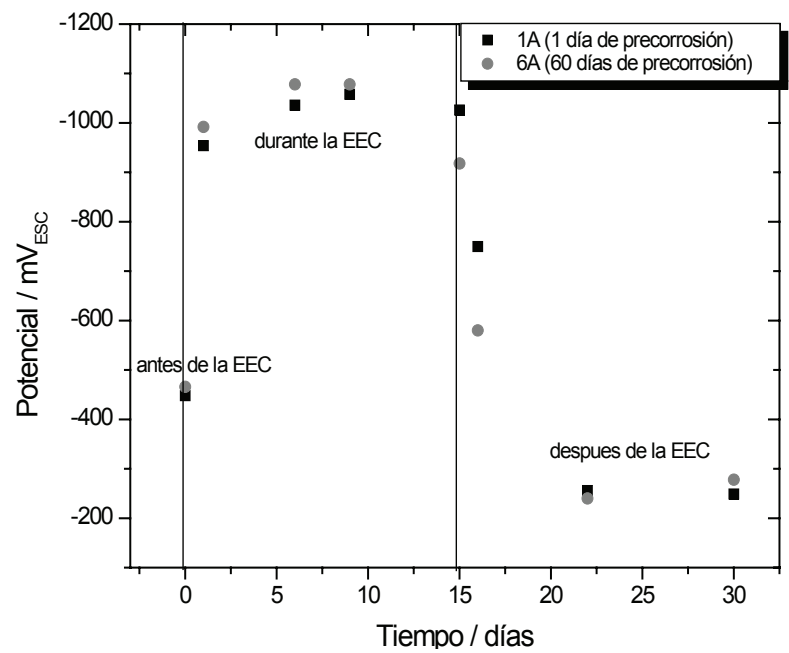

das con $2 \% \mathrm{NaCl}$. Como se puede apreciar antes de iniciar con la extracción, los valores de las armaduras se situaban en una zona de alta probabilidad de corrosión $(-450 \mathrm{mV})$. Durante el tratamiento la armadura de acero presentó una elevada polarización de $-1080 \mathrm{mV}$. Una vez que las varillas fueron desconectadas de la fuente de corriente, se realizaron nuevamente mediciones de los potenciales; sin embargo, los primeros días estos valores no se estabilizaron debido a la alta polarización que tuvieron durante los sesenta días de extracción. El potencial de las varillas se estabilizó después de 40 días. Estos potenciales ya estables, situaron a las armaduras en una zona incierta de corrosión, como lo marca la norma ASTM 876.

Con respecto a las varillas sin EEC y contaminadas con cloruros, los valores de los potenciales fueron constantes de $-450 \mathrm{mV}$. Con estos resultados se podría pensar que la extracción logró bajar los potenciales de las varillas a una zona menos probable de corrosión, pero no se tiene la certeza de que las varillas lograran su despasivación.

En las figuras $5 \mathrm{a}$ y $5 \mathrm{~b}$ se presentan las densidades de corrosión de las armaduras de acero en función de los tiempos de EEC. La Figura 5a para muestras precorroidas durante 1 día y la Figura $5 \mathrm{~b}$ para muestras precorroidas durante sesenta días. Se observa que antes de iniciar con los tratamientos las velocidades de corrosión de los refuerzos son cercanos a $1 \mu \mathrm{A} / \mathrm{cm}^{2}$, de acuerdo con estos valores, estos $\mathrm{i}_{\text {corr }}$ resultan muy peligrosos en las estructuras de concreto armado, ya que pueden provocar grietas en los recubrimientos de concreto para

b)

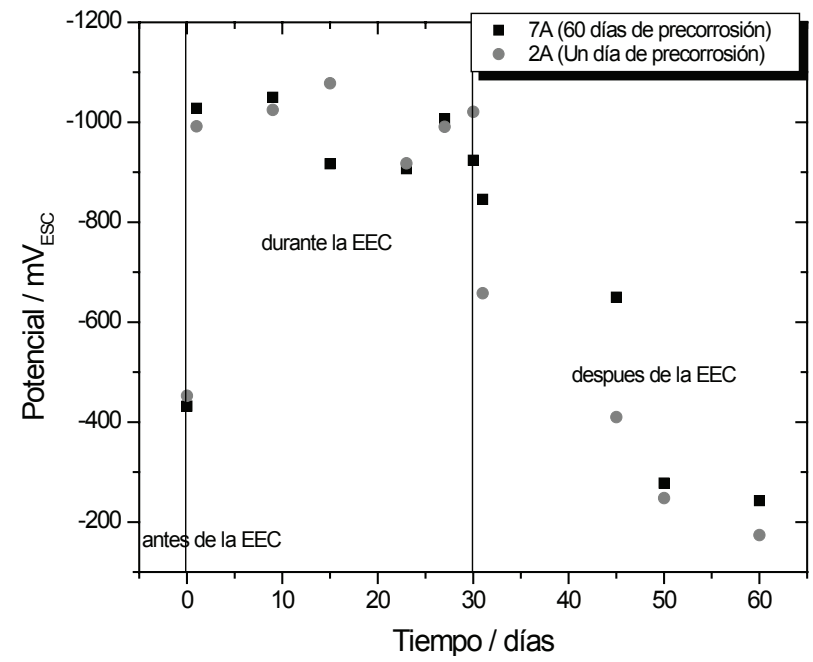

Figura 3. Evolución de los $\mathrm{E}_{\text {corr }}$ antes, durante y después de las EEC. En tiempos de 15 y 30 días de extracción, respectivamente (3a y $3 b)$ 


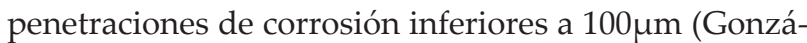
lez et al., 1993).

En la figura 5a se muestra que estas intensidades de corrosión en los electrodos disminuyeron hasta alcanzar valores de $0.1 \mu \mathrm{A} / \mathrm{cm}^{2}$, después de efectuar extracciones en diferentes tiempos y previos a una precorrosión de un día. Para estos $i_{\text {corr, }}$ los electrodos se ubican dentro de los límites atribuibles al estado pasivo (González et al., 1994; Broomfield, 1991; Clear, 1990). Así se manifiesta que las armaduras de acero pueden despasivarse con la EEC, si el grado de precorrosión es incipiente (1 día de precorrosión).

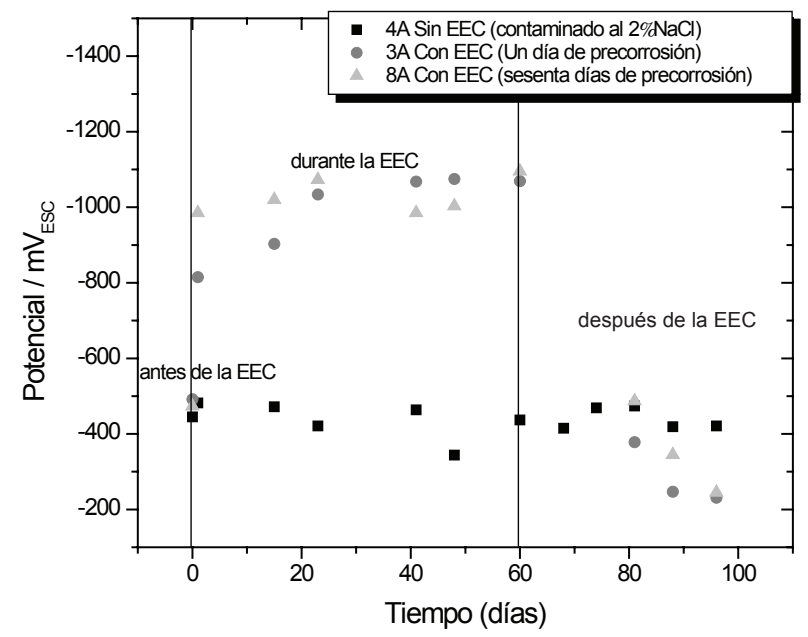

Figura 4. Evolución de los $\mathrm{E}_{\text {corr }}$, sin y con extracciones de 60 días

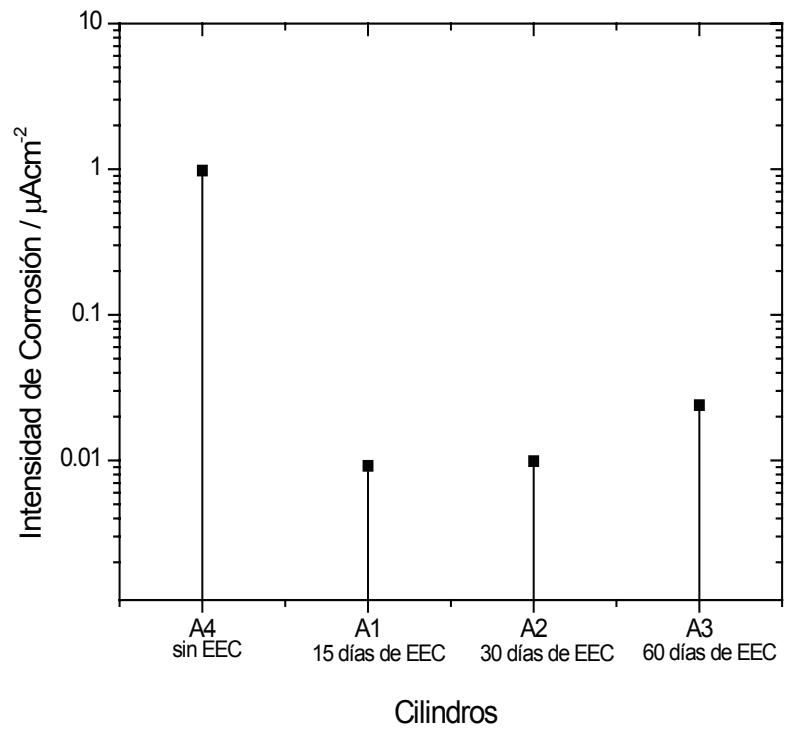

Figura 5a. $I_{\text {corr }}$ contra tiempos de EEC, previamente expuestos a un día de precorrosión
En la figura $5 b$ se observa que los electrodos con sesenta días de precorrosión y después de efectuar extracciones en diferentes tiempos, las densidades de corrosión se situaron en valores de $0.1 \mathrm{a} 1 \mu \mathrm{A} / \mathrm{cm}^{2}$, siendo un estado activo y muy peligroso, de acuerdo con los riesgos señalados en el párrafo precedente. Esto nos permite decir que una vez que los electrodos se someten a tiempos prolongados de precorrosión (60 días), la EEC no es capaz de repasivar las varillas de acero, aun con diferentes tiempos de tratamiento. Cabe señalar que los valores de los $i_{\text {corr }}$ que se muestran después de las extracciones, fueron calculados con los potenciales ya estables.

En la tabla 2 se presentan los análisis puntuales de porcentaje en peso de los $\mathrm{Cl}^{-}$remantes en cilindros de mortero, después de realizar extracciones de 15, 30 y 60 días. Se realizaron 3 tomas en cada posición y se obtuvieron los promedios de estos puntos, desde la superficie del mortero hacia la varilla, con avances de $1 \mathrm{~cm}$. Los análisis muestran que existe un incremento de los cloruros retenidos cerca de la varilla para las tres muestras y una disminución de cloruros en la superficie del mortero. El avance no es constante, hay una mayor cantidad de cloruros removidos en la superficie del mortero que en el interior de la varilla, después de realizarse la EEC. Esto puede deberse a que en el interior del cilindro (cerca de la varilla) la densidad de corriente es más baja y por lo tanto, la migración de los iones cloruro es más lenta. En otras palabras, la cantidad de cloruros retenidos después de efectuar la EEC varía a lo largo de la sección trasversal del mortero debido a que las condi-

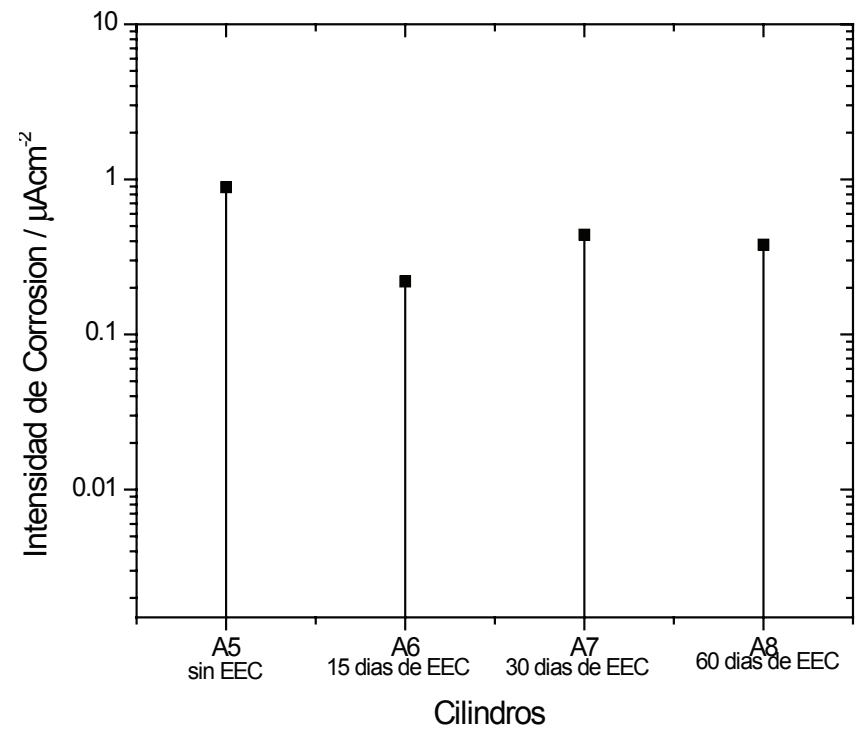

Figura 5b. $\mathrm{I}_{\text {corr }}$ contra tiempos de EEC, previamente expuestos a sesenta días de precorrosión 
ciones electroquímicas cambian. Estos análisis puntuales se realizaron mediante el uso de un SEM (microscopio electrónico de barrido).

Tabla 2. Análisis puntuales de \% wt de $\mathrm{Cl}^{-}$en cilindros de mortero, después de realizar extracciones de 15, 30 y 60 días

\begin{tabular}{|c|c|c|c|c|}
\hline \multirow[b]{2}{*}{ Núm. } & \multirow[b]{2}{*}{$\begin{array}{l}\text { EEC } \\
\text { (días) }\end{array}$} & \multicolumn{3}{|c|}{$\begin{array}{c}\text { DESDE LA SUPERFICIE HACIA LA } \\
\text { VARILLA }\end{array}$} \\
\hline & & $\begin{array}{c}1 \mathrm{~cm} \\
\% \text { wt de Cl- }\end{array}$ & $\begin{array}{c}2 \mathrm{~cm} \\
\% \text { wt de Cl- }\end{array}$ & $\begin{array}{c}3 \mathrm{~cm} \\
\% \text { wt de Cl- }\end{array}$ \\
\hline $1 \mathrm{~A}$ & 15 & 0.15 & 0.23 & 0.53 \\
\hline $2 \mathrm{~A}$ & 30 & 0.19 & 0.27 & 0.41 \\
\hline $3 \mathrm{~A}$ & 60 & 0.18 & 0.31 & 0.43 \\
\hline
\end{tabular}

\section{Conclusiones}

De acuerdo con los resultados obtenidos en esta investigación se concluye lo siguiente:

- La cantidad de Cl- removidos del mortero es directamente proporcional al tiempo de la EEC.

- El grado de precorrosión no afecta a la eficiencia de la EEC.

- Los refuerzos de acero presentan una polarización ( $\approx-1000 \mathrm{mV})$, esto posiblemente por la EEC. Para tiempos más prolongados de las extracciones, es mayor el tiempo de espera para estabilizar los $E_{\text {corr }}$.

- Las armaduras de acero pueden despasivarse con la EEC, si el grado de precorrosión es incipiente (1 día de precorrosión). Sin embargo, si los electrodos se someten a tiempos prolongados de precorrosión (60 días), la EEC no es capaz de repasivar a los refuerzos de acero.

- Hay una mayor cantidad de cloruros removidos en la superficie del mortero, que en el interior de la varilla después de realizarse la EEC. Esto puede deberse a que en el interior del cilindro (cerca de la varilla) la densidad de corriente es más baja y por lo tanto la migración de los iones cloruro más lenta.

\section{Referencias}

Biezma M.V., San Cristobal J.R. Is the Cost of Corrosion Really Quantifiable. Corrosion, volumen 62 (número 12), 2006: 10511055.

Browne R.D., Geoghegan M.P., Baker A.F. Analysis of Structural Condition from Durability Results, Corrosion of Reinforcement in Concrete Construction, ed. AP, Londres, Crane SCI, 1983, pp. 193-222.

Broomfiel J.P. Life Prediction of Corrodible Structures, en: NACE Symposium, Cambridge, septiembre 1991, artículo 14.
Clear K.C. 3LP Package: Tests Procedures, Data Analysis and General Information, julio 1990, Appendix, p.9.

Fajardo G., Escadeillas G. y Arliguie G. Electrochemical Chloride Extraction (ECE) from Steel-Reinforced Concrete Specimens Contaminated by Artificial Sea-Water. Corrosion Science, volumen 48, 2006:110-125.

Green W.K., Lyon S.B., Scantlebury J.D. Electrochemical Changes in Chloride-Contaminated Reinforced Concrete Following Cathodic Polarization, Corrosion Science (números 5-8), 1993: 1627-1631

González J.A., Andrade C., Rodriguez P., Alonso C., Feliu S. Effect Current on the Degradation of Reinforced Concrete Structures, en: Progress in the Understanding and Prevention of Corrosion, Instituto de Materiales y la Sociedad Española de Química Industrial, Reino Unido, 1993, pp. 629-633.

González J.A., Benito M., Bautista A., Ramírez. Inspección y diagnostico de las estructuras de hormigón armado. Rev. Metal Madrid, volumen 30 (número 5), 1994: 271-282.

Lankard et al. Neutralization of Chloride in Concrete. Report No. FHWA-RD-7660, 1975, pp. 1-143.

Morrison G.L., Virmani Y.P. Stratton: Chloride Removal and Monomer Impregnation of Bridge Deck Concrete by Electro-Osmosis, Report No. FHWA-KS-RD-74-1, 1976, pp. 1-41.

Orellan J.C., Escadeillas G., Arliguie G. Electrochemical Chloride Extraction: Efficiency and Side Effects. Cement and Concrete Research, volumen 34, 2004: 227-234.

Page C.L., Y.-Treadaway K.W.J. Aspects of Electrochemistry of Steel i Concrete. Nature, volumen 297 (número 5862), 1982: 109. Stern M., Geary A.L. Electrochemical Polarization. I: A Theoretical Analysis of the Shape of Polarization Curves. J. Electrochemical. Soc., volumen 104, 1957: 56-63.

\section{Este artículo se cita:}

\section{Citación Chicago}

Rodríguez-Reyna, Sandra Luz, Juana María Miranda-Vidales, Dora Erika Espericueta-González, Citlalli Gaona-Tiburcio. Results of an ECE Varying Degrees of Corrosion and Time of Application of the Technique. Ingeniería Investigación y Tecnología, XIII, 04 (2012): 425-431.

\section{Citación ISO 690}

Rodríguez-Reyna S.L., Miranda-Vidales J.M., Espericueta-González D.E., Gaona-Tiburcio C. Results of an ECE Varying Degrees of Corrosion and Time of Application of the Technique. Ingeniería Investigación y Tecnología, volumen XIII (número 4), octubre-diciembre 2012: 425-431. 


\section{Semblanza de los autores}

Sandra Luz Rodríguez-Reyna. Obtuvo el grado de ingeniero mecánico administrador por la Universidad Autónoma de San Luis Potosí en 2002, asimismo, el grado de maestría en metalurgía e ingeniería de materiales por el Instituto de Metalurgía en 2006. Su colaboración en el área de ciencias ha sido dentro y fuera del país con investigadores de reconocimiento internacional. Desde 2011 es profesora investigadora visitante de la Facultad de Ingeniería de la UASLP, impartiendo los cursos de mecánica de materiales y materiales eléctricos. Sus áreas de interés son: corrosión en las estructuras de concreto armado y técnicas de rehabilitación. Actualmente es jefe de Laboratorio de Materiales del Área Mecánica y Eléctrica en la Universidad Autónoma de San Luis Potosí.

Juana María Miranda-Vidales. Realizó la licenciatura en química y la maestría en metalurgía e ingeniería de materiales en la UASLP. El doctorado lo obtuvo en ciencia y tecnología de materiales por la Universidad Complutense de Madrid, España. Trabajó en la industria minera y metal mecánica en el área de control de calidad. Cuenta con más de 20 artículos internacionales en revistas indexadas y más de 30 publicaciones en memorias en extenso en congresos nacionales e internacionales. En el área de docencia ha impartido cursos en licenciatura, maestría y doctorado dentro de la UASLP. Actualmente pertenece al SNI nivel uno.

Dora Erika Espericueta-González. Obtuvo el grado de ingeniero mecánico administrador en la UASLP en 1997. De 1997 a 2004, desempeñó funciones en la empresa Cummins S. de R.L. de C.V., en las áreas de mercadotecnia y herramientas. Obtuvo el grado de maestra en el Instituto de Metalurgia en 2007. Actualmente colabora con el cuerpo académico de materiales de la Facultad de Ciencias-UASLP y se desempeña como profesora investigadora impartiendo materias de calidad, probabilidad e inferencia estadística e ingeniería de materiales. Su línea de investigación es la síntesis y caracterización de materiales.

Citlalli Gaona-Tiburcio. Obtuvo el grado de ingeniero metalurgista en la Universidad Autónoma Metropolitana Azcapotzalco en 1991. Obtuvo el grado de maestría en la Facultad de Química de la UNAM y en 1999 el doctorado en ciencias de materiales por el Centro de Investigación en Materiales Avanzados (CIMAV). De 1995 a 2011 se desempeñó como investigadora en dicha institución, impartiendo las materias de corrosión, técnicas electroquímicas y mecanismos de corrosión en el programa de posgrado. Pertenece al Sistema Nacional de Investigadores (SNI). A partir de octubre de 2011 se incorporó al CIIIA de la Facultad de Ingeniería Mecánica y Eléctrica de la Universidad Autónoma de Nuevo León. 